\title{
Dificultades en la Accesibilidad Web de las Universidades Españolas de acuerdo a la Norma WCAG 2.0
}

\author{
Antonio Chacón-Medina*, Helena Chacón-López**, M. Dolores López-Justicia**, Carolina Fernández-Jiménez** \\ * Grupo APESDI (HUM-846), Departamento de Didáctica y Organización Escolar. Universidad de Granada (España). \\ ** Grupo APESDI (HUM-846). Departamento de Psicología Evolutiva y de la Educación. Universidad de Granada (España). \\ Correo-e: chacon@ugr.es
}

Recibido: 27-07-2012; 2a version: 13-03-2013; Aceptado: 21-03-2013.

Cómo citar este artículo/Citation: Chacón-Medina, A.; Chacón-López, H.; López-Justicia, M. D.; Fernández-Jiménez, C. (2013). Dificultades en la Accesibilidad Web de las Universidades Españolas de acuerdo a la Norma WCAG 2.0. Revista Española de Documentación Científica, 36(4):e025. doi: http://dx.doi.org/10.3989/redc.2013.4.1009

Resumen: El propósito del estudio ha sido conocer posibles limitaciones en el nivel de accesibilidad de las páginas principales y de estudiantes, de los sitios web de 74 Universidades españolas, para lo cual se ha utilizado la herramienta TAW, que permite analizar, de acuerdo a la norma WCAG 2.0, la existencia de barreras en ellas. Después de revisar automática y manualmente los Principios, Pautas y Criterios de conformidad de la norma, en sus niveles de prioridad $A$ y $A A$, un análisis porcentual constata que ni una sola de las universidades los supera en su totalidad en ninguna de las dos páginas analizadas, aunque la mejor situada en accesibilidad es la de Granada en ambas páginas, seguida de la de Alicante. Se concluye recomendando hacer chequeos y ajustes para resolver estos problemas y facilitar la accesibilidad web.

Palabras clave: Accesibilidad Web; WCAG 2.0; TAW; discapacidad; universidad.

\section{The State of Web Accessibility in Spanish Universities according to WCAG 2.0}

Abstract: Abstract: The purpose of the study has been to learn of possible limitations in the level of accessibility of 74 Spanish universities' web sites. The tool, TAW, was used to detect the existing barriers in each web, based on the WCAG 2.0 regulation. After an automatic and manual review of the principles, guidelines and criteria of priority levels $A$ and $A A$, a percentage analysis showed that none of the universities surpassed these aspects entirely for either of the two web pages analysed, although the university with the best accessibility on both web pages was the University of Granada, followed by the University of Alicante. The study concludes by recommending revisions and adjustments to resolve these problems and to facilitate web accessibility.

Keywords: Web accessibility; WCAG 2.0; TA; disability; university.

Copyright: (c) 2013 CSIC. Este es un artículo de acceso abierto distribuido bajo los términos de la licencia Creative Commons Attribution-Non Commercial (by-nc) Spain 3.0. 


\section{INTRODUCCIÓN}

La red se ha convertido en el momento actual en un elemento capaz de favorecer la integración social y laboral de las personas con discapacidad, desde el momento en que facilita la comunicación, el acceso y procesamiento de la información, el desarrollo cognitivo, la participación en ámbitos educativos y de ocio y el desempeño de actividades laborales (Marquès, 2004). Por ello, facilitar el acceso a todos los recursos que ofrece Internet debe ser un objetivo fundamental, requiriendo el logro de un diseño web accesible.

La accesibilidad ha sido descrita como un mecanismo que asegura la igualdad de oportunidades (Harper y Yesilada, 2008), asumiendo que una página o sitio web es accesible cuando se diseña para que sus contenidos y servicios estén disponibles para cualquier usuario, independientemente de sus condiciones personales, o contexto de navegación (Henry, 2002), haciendo más fácil tanto el acceso, como el uso del recurso (Hassan y Martín, 2004); sin embargo, se ha constatado (Harper y Yesilada, 2008) que las personas con discapacidad siguen teniendo dificultades para acceder a la web.

\section{ACCESIBILIDAD A LOS CONTENIDOS EN LA WEB. LA NORMA WCAG $\mathbf{2 . 0}$}

La accesibilidad web responde a recomendaciones técnicas, establecidas fundamentalmente por la World Wide Web Consortium (W3C), cuyo objetivo es desarrollar estándares web, a través de la creación de directrices y estrategias (Greeff y Kotzé, 2009). Desde ella, la WAI (Web Accessibility Initiative), propone una guía para hacer más accesibles las webs a personas con discapacidad, surgiendo en su seno el "Grupo de Trabajo de las Pautas de Accesibilidad al Contenido en la Web" (WCAG WG - Web Content Accessibility Guidelines Work Group).

Las WCAG 2.0 constituyen en la actualidad el referente normativo en materia de accesibilidad web. Estas normas, publicadas en 2008 por el W3C (WAI), suponen una continuación y mejora de las WCAG 1.0, al ser más fáciles de utilizar, entender (W3C, 2006) y susceptibles de aplicación en cualquier tecnología. Entre sus ventajas también destaca el ser más precisas y verificables, tanto si usamos evaluación automática (software que analiza el código de una página web, devolviendo una serie de resultados), como con revisión manual mediante evaluadores humanos (Pinto, 2011).

La norma WCAG 2.0 se estructura en tres niveles fundamentales: principios, pautas y criterios de conformidad (Fundación Sidar, 2009). Estos tres niveles se complementan con una serie de técnicas recomendadas para obtener éxito en los diferentes criterios. En el nivel más alto contempla cuatro "principios", los cuales contienen 12 "pautas", y cada una de éstas se compone de un número determinado de "criterios de conformidad" (61 en total), que constituyen los ítems a verificar. En los criterios se definen tres "niveles de conformidad": A (el más bajo), AA y AAA (el más alto). Esta estructura se concreta como sigue:

Principio 1. Perceptibilidad: el contenido informativo, así como todos los elementos de la interfaz deben presentarse a los usuarios de manera que puedan percibirlos. Este principio contiene cuatro pautas, que se ocupan de:

- Pauta 1.1: Proporcionar alternativas textuales para cualquier contenido no textual. Contiene un sólo criterio de conformidad de nivel $A$.

- Pauta 1.2: Proporcionar alternativas para el contenido multimedia. Está formada por nueve criterios (tres de nivel $A$, dos de nivel AA y cuatro de nivel AAA).

- Pauta 1.3: Presentación del contenido de distintas formas, sin perder información ni estructura. Tiene tres criterios de nivel $A$.

- Pauta 1.4: Facilitar a los usuarios ver y escuchar el contenido. Contiene dos criterios de nivel $A$, tres de nivel $A A$ y cuatro de nivel AAA.

Principio 2. Operabilidad: las funcionalidades del contenido deben estar diseñadas para poder ser realizadas a través de un teclado, o una interfaz de éste. Formado por cuatro pautas:

- Pauta 2.1: Todas las funciones deben estar disponibles mediante teclado. Contiene dos criterios de nivel $A$ y uno AAA.

- Pauta 2.2: Permanencia del contenido durante suficiente tiempo para leerlo y usarlo. Formada por dos criterios de nivel $A$ y tres de nivel AAA.

- Pauta 2.3: Cuidado con formas que puedan provocar ataques epilépticos, espasmos o convulsiones. Tiene un criterio de nivel $A$ y uno AAA.

- Pauta 2.4: Ayuda para navegar, localizar contenidos y determinar dónde se encuentra. Formada por cuatro criterios de nivel $A$, tres de nivel $A A$ y tres de nivel AAA.

Principio 3. Comprensibilidad: El contenido y los controles de la interfaz de usuario han de ser comprensibles. Su contenido se estructura en torno a tres pautas:

- Pauta 3.1: El contenido debe ser legible y comprensible. Contiene un criterio de nivel $A$, uno $A A$ y tres $A A A$.

- Pauta 3.2: La apariencia y la operabilidad de las páginas Web deben ser pre- 
decibles. Formada por dos criterios de nivel $A$, dos $A A$ y uno de nivel AAA.

- Pauta 3.3: Ayuda a los usuarios a evitar y corregir errores en la entrada de datos. Tiene dos criterios en cada uno de los niveles ( $A, A A$ y $A A A)$.

Principio 4. Robustez: El contenido debe estar diseñado para que funcione con las diferentes tecnologías, incluidas las ayudas técnicas. Está formado por una sola pauta que contiene dos criterios de nivel A.

\section{DIFICULTADES EN LA ACCESIBILIDAD A LA WEB}

Teniendo en cuenta las referidas normativas, se han formulado diferentes leyes internacionales y nacionales tratando de garantizar la igualdad de oportunidades en relación a la tecnología de la información y la comunicación (Ribera, Térmens y Frías, 2009; Rocha, Cobo y Alonso, 2011). Pero a pesar del soporte legal existente, su incumplimiento está propiciando desde hace unos años la aparición de una nueva forma de exclusión social (Alba, Ruiz y Zubillaga, 2003); observándose que las personas con discapacidad (al igual que las personas mayores), aun disponiendo de los medios materiales y de la formación adecuada para hacer uso de la red, encuentran dificultades. Situación observada, igualmente, en la educación superior (Alba y otros, 2003; Ribera y otros, 2009; Térmens y otros, 2003; Térmens, 2002; Toledo, 2001). No obstante, en este contexto un diseño accesible debería ser considerado prioritario, porque los estudios universitarios se encuentran en el último eslabón formativo y porque la presencia de alumnado con discapacidad va en progresivo aumento. Este hecho puede provocar que algunos de estos estudiantes experimenten serias dificultades para utilizar la web, debido a la combinación de diferentes tipos de barreras sobre las que poco se ha investigado.

En España, varios trabajos han analizado el nivel de accesibilidad y la forma en que se presenta la información en las distintas web de las universidades, tanto públicas como privadas (Alba y otros, 2003; Ribera y otros, 2009; Rocha y otros, 2011; Rodríguez y otros, 2006; Térmens y otros, 2003; Térmens, 2002; Toledo, 2001), de acuerdo con la norma WCAG 1.0, constatándose un bajo grado de cumplimiento y seguimiento de las recomendaciones internacionales y nacionales para favorecer la accesibilidad de los sitios web. La detección de estas dificultades ha sido posible gracias a la aparición de diferentes instrumentos, entre los más utilizados para realizar esta función, de acuerdo con las WCAG 2.0, se encuentra el Test de Accesibilidad Web (TAW) (Serrano, Moratilla y Olmeda, 2010).

\section{OBJETIVOS DEL ESTUDIO}

Dada la repercusión que puede tener el incumplimiento de la normativa en el acceso a la web de las personas con discapacidad (Alba y otros, 2003; Ribera y otros, 2009; Térmens y otros, 2003; Térmens, 2002; Toledo, 2001), nos hemos marcado como principal objetivo en el presente trabajo: analizar el nivel de accesibilidad de las páginas principales y de estudiantes de los sitios web de las Universidades españolas (tanto públicas como privadas) de acuerdo con la norma actual, para conocer si se mantienen los fallos detectados en estudios previos o se han implementado soluciones. Se pretende, igualmente, comprobar cuáles son los problemas o errores que se presentan con mayor frecuencia y el nivel de cumplimiento de la normativa vigente en materia de accesibilidad web.

\section{MATERIAL Y MÉTODO}

\section{Muestra}

La muestra del estudio ha estado constituida por la totalidad de las Universidades españolas: 54 públicas y 20 privadas.

\section{Instrumento}

El software usado para el estudio es el TAW, herramienta desarrollada por la Unidad de Accesibilidad Web de la Fundación CTIC (Centro Tecnológico de la Información y la Comunicación), con la colaboración del CEAPAT (García, 2006).

Entre las herramientas de la familia TAW, hemos usado la denominada "TAW3 Online (WCAG 2.0)", que permite revisar la accesibilidad de una determinada URL, bajo la norma WCAG 2.0; generando un informe HTML. El software ofrece la posibilidad de personalizar el análisis eligiendo el nivel de exigencia ( $A, A A$ y $A A A)$ y de revisar las diferentes tecnologías que pueden incorporar los sitios web (HTML, CSS y Javascript). El informe muestra cuatro pestañas (Fundación CTIC, s.f.):

- Resumen: visión general con el total de errores (correcciones necesarias), advertencias (deben revisarse manualmente) y puntos no verificados (que requieren un análisis manual completo).

- Vista marcada: muestra sobre la página web, las incidencias detectadas.

- Detalle: informa del número de línea de código que contiene la incidencia y enlaza al sitio del $W 3 C$ en el que se alude a las técnicas relacionadas.

- Listado: resumen agrupado por cada uno de los principios básicos, en formato de tabla, que indica el resultado obtenido en cada una de las pautas y criterios.

Posteriormente se ha utilizado el complemento "Web Developer (v.1.1.9)" (extensión para navegadores web basados en Mozilla Firefox), que proporciona una serie de utilidades de edición y 
depuración para desarrolladores web. Esta herramienta se presenta como una barra del navegador con menús extensibles, desde los que es posible acceder a los distintos elementos de la página para efectuar su comprobación.

\section{Procedimiento}

Se parte de un estudio previo (tipo encuesta mediante el software "LimeSurvey") con una muestra de 745 estudiantes, de distintas titulaciones, de la Facultad de Ciencias de la Educación de la Universidad de Granada (UGR), realizado por los autores durante el mes de octubre de 2011; del que se desprende que las dos páginas usadas de forma directa para entrar en el sitio de esta universidad son la página Principal y su enlace a la de Estudiantes. Aunque los resultados no pueden extenderse sin más al resto de universidades, no obstante, orientaron en el procedimiento a seguir en el análisis siguiente. Posteriormente, se constató, mediante el acceso al sitio web, que el 91,89\% (68 de 74) de las universidades españolas cuentan con enlaces directos en su página principal a páginas de "Estudiantes" o "Alumnos"; lo que permitió elegir para el análisis estas dos páginas en cada universidad. En el 8,11\% restante de casos se analizaron la Principal y la de "Estudios". El motivo de centrar la atención en los alumnos se debe a que su número es muy superior al resto de colectivos.

Seguidamente, se inició el proceso de análisis, procediéndose a realizar el volcado manual de datos en una plantilla de una hoja de cálculo (Microsoft Excel, v. 14.2.2 para Mac). El tratamiento de la información obtenida consistió, fundamentalmente, en cálculos porcentuales sobre los diferentes datos aportados por el programa.

Para llevar a cabo el análisis automático, se proporcionó al programa la URL de las páginas principales y de estudiantes del sitio web de cada una de las universidades, analizándose las páginas de cada uno de ellos; seleccionando como opción el "nivel de exigencia" AA, y como "tecnologías soportadas", el código HTML y las hojas de estilo CSS. No se seleccionó el nivel AAA (máxima exigencia), ya que desde el $W 3 C$ recomiendan, como política general, no exigirlo porque en algunos contenidos no es posible satisfacer todos sus criterios de conformidad (Fundación Sidar, 2009).

A continuación se completó el análisis con los resultados de la revisión manual.

El análisis automático de los diferentes sitios web se realizó durante los meses de noviembre y diciembre de 2011; mientras que la revisión manual de los diferentes errores detectados, y de las páginas de imposible comprobación automática, se hizo desde enero a mayo de 2012

Del análisis automático, llevado a cabo con TAWWCAG 2.0 Beta, extraemos resultados acerca de: 1 . Criterios de conformidad que se cumplen; 2 .
Problemas o errores encontrados en ellos; 3. Criterios que requieren de revisión manual; 4. Páginas que no se han podido analizar; 5. Páginas a las que no procede aplicar los criterios de análisis, por no contener el tipo de elementos que se analizan en ellos.

Para la revisión manual partimos del listado que ofrece la herramienta en la pestaña "Detalle", en la que se señalan los números de línea que conviene revisar, con la ayuda del complemento "Web Developer". Durante este proceso, cuando alguno de los criterios para una página presentaba el primer error se detenía el análisis, por tanto en esta revisión no se ha contabilizado el número de errores hallados, sino el cumplimiento o no del criterio.

\section{RESULTADOS}

Se detallan primeramente los resultados porcentuales del análisis automático de las páginas:

1. Conjunto de criterios en los que TAW de manera automática determina que "no se han encontrado errores", y el porcentaje de universidades que los satisfacen (Tabla I). En el grupo de criterios de nivel AA sólo aparece uno (el 1.4.4) que se cumple en parte del conjunto de universidades $(14,86 \%$ 11 universidades), en ambas páginas, aunque no son las mismas.

En el bloque de nivel $A$, encontramos el mayor número de criterios que se cumplen parcialmente (8 en la página principal y 9 en la de estudiantes). El nivel de cumplimiento en la página principal, oscila entre el 72,97\% (54 universidades) del criterio 3.1 .1 y el $1,35 \%$ (1 universidad) del criterio 1.1.1; mientras que en la página de estudiantes, se sitúa en el 71,62 \% (53 universidades), en el criterio 3.1 .1 , y el $1,35 \%$ del 1.1 .1 y del 4.1 .2 (1 universidad).

2. Conjunto de errores o problemas de accesibilidad encontrados por TAW (mediante análisis automático) en las diferentes páginas: aparecen errores en tres criterios del Principio de "Perceptibilidad", tres en el de "Operabilidad" (aunque los ítems 2.1 .2 y 2.4 .2 realmente no sean muy representativos), tres en el de "Comprensión" y dos en el de "Robustez". Observamos, por tanto, errores en todos los principios (Tabla II).

3. Criterios para los se requiere verificación manual (Tabla III) para asegurarse de su cumplimiento, ya que el programa no lo hace de forma segura. En esta situación también encontramos criterios de todos los principios. El porcentaje total de páginas a revisar parcialmente de forma manual es del $38,19 \%$ en el caso de la principal, y del $41,76 \%$ en la de estudiantes.

4. El programa detecta un alto número de criterios en los que da como resultado "Imposible realizar comprobación automática" (Tabla IV), por lo que posteriormente se realizará también una re- 
Tabla I. Ítems que, según el análisis automático de TAW, se cumplen en su totalidad o en parte, número de universidades en las que se cumple y porcentaje

\begin{tabular}{lcccc}
\hline & \multicolumn{2}{c}{ Principal } & \multicolumn{2}{c}{ Estudiantes } \\
\hline \multicolumn{1}{c}{ Ítem } & Cumple & \% & Cumple & $\%$ \\
\hline 1.1.1 Contenido no textual & 1 & 1,35 & 1 & 1,35 \\
\hline 1.3.1 Información y relaciones & 18 & 24,32 & 14 & 18,92 \\
\hline 1.3.2 Secuencia significativa & 3 & 4,05 & 4 & 5,41 \\
\hline 1.4.4 Cambio de tamaño de texto & 11 & 14,86 & 11 & 14,86 \\
\hline 2.4.4 Propósito de los enlaces (en contexto) & 21 & 28,38 & 13 & 17,57 \\
\hline 3.1.1 Idioma de la página & 54 & 72,97 & 53 & 71,62 \\
\hline 3.3.2 Etiquetas o instrucciones & 41 & 55,41 & 37 & 50 \\
\hline 4.1.1 Procesamiento & 7 & 9,46 & 5 & 6,76 \\
\hline 4.1.2 Nombre, función, valor (interfaz usuario) & & & 1 & 1,35 \\
\hline
\end{tabular}

Tabla II. Ítems en los que TAW, realizando el análisis automático, ha encontrado errores (número de universidades, porcentaje de las mismas y número de ellos)

\begin{tabular}{ccccccc}
\hline & & Principal & & \multicolumn{2}{c}{ Estudiantes } \\
\hline Ítem & Univ. & \% & Errores & Univ. & \% & Errores \\
\hline 1.1 .1 & 60 & 81,08 & 354 & 56 & 75,58 & 257 \\
\hline 1.3 .1 & 51 & 68,92 & 359 & 53 & 71,62 & 504 \\
\hline 1.4 .4 & 41 & 55,41 & 433 & 43 & 58,11 & 317 \\
\hline 2.1 .2 & & & & 1 & 1,35 & 1 \\
\hline 2.4 .2 & & & & 2 & 2,70 & 218 \\
\hline 2.4 .4 & 39 & 52,70 & 338 & 40 & 54,05 & 21 \\
\hline 3.1 .1 & 18 & 24,32 & 18 & 20 & 27,03 & 16 \\
\hline 3.2 .2 & 16 & 21,62 & 19 & 15 & 20,27 & 34 \\
\hline 3.3 .2 & 23 & 31,08 & 30 & 25 & 33,78 & 445 \\
\hline 4.1 .1 & 47 & 63,51 & 632 & 45 & 60,81 & 50 \\
\hline 4.1 .2 & 31 & 41,89 & 56 & 31 & 41,89 & 2 \\
\hline
\end{tabular}

Tabla III. Número de páginas en las que TAW recomienda la revisión manual de algunos criterios, porcentaje sobre el total de páginas a analizar en cada Principio en la página Principal y en la de Estudiantes

\begin{tabular}{|c|c|c|c|c|c|c|c|c|c|}
\hline & \multirow[b]{2}{*}{ Pauta } & \multicolumn{4}{|c|}{ Principio 1} & \multicolumn{2}{|c|}{ Principio 2} & \multirow{2}{*}{$\begin{array}{c}\begin{array}{c}\text { Principio } \\
\mathbf{3}\end{array} \\
3.3\end{array}$} & \multirow{2}{*}{$\begin{array}{c}\begin{array}{c}\text { Principio } \\
\mathbf{4}\end{array} \\
4.1\end{array}$} \\
\hline & & 1.1 & 1.2 & 1.3 & 1.4 & 2.1 & 2.4 & & \\
\hline \multirow{2}{*}{ Principal } & No & 13 & 30 & 71 & 28 & 23 & 271 & 191 & 21 \\
\hline & $\%$ & 17,57 & 8,11 & 47,97 & 18,92 & 15,54 & 61,04 & 86,04 & 14,19 \\
\hline \multirow{2}{*}{ Estudiantes } & No & 17 & 15 & 72 & 20 & 21 & 272 & 177 & 24 \\
\hline & $\%$ & 22,97 & 4,05 & 48,75 & 27,03 & 28,38 & 61,26 & 79,73 & 32,43 \\
\hline
\end{tabular}


visión manual de los mismos. Hay 13 criterios en la página principal y 12 en la de estudiantes en los que el programa no puede realizar el análisis en ninguna de las universidades. Teniendo en cuenta el total de criterios en los niveles A y AA (38), y el número de ellos que aparecen como "imposibles de analizar" (Tabla IV), el porcentaje sobre el que será necesario hacer revisión es del 43,50 \%.
5. Criterios en los que no procede la realización del análisis, por no contener la página los elementos susceptibles de análisis, fundamentalmente contenidos de carácter multimedia. Encontramos 12 criterios (31,58 \%), en los niveles de prioridad contemplados (Tabla V), si bien no inciden con la misma frecuencia en todas la universidades.

Tabla IV. Criterios de cada Principio (P1, P2, P3 y P4) en los que TAW da como resultado "Imposible realizar comprobación automática", porcentaje y número de universidades

\begin{tabular}{|c|c|c|c|c|c|}
\hline \multirow[b]{2}{*}{ Principio } & \multirow[b]{2}{*}{ Criterio } & \multicolumn{2}{|c|}{ Principal } & \multicolumn{2}{|c|}{ Estudiantes } \\
\hline & & $\%$ & $n^{0}$ & $\%$ & $n^{0}$ \\
\hline \multirow{4}{*}{ P1 } & 1.3.3- Características sensoriales & 100 & 74 & 100 & 74 \\
\hline & 1.4.1- Uso del color & 100 & 74 & 100 & 74 \\
\hline & 1.4.3- Contraste (texto, imagen, logotipos) & 100 & 74 & 100 & 74 \\
\hline & 1.4.5- Imágenes de texto & 100 & 74 & 100 & 74 \\
\hline \multirow{9}{*}{ P2 } & 2.1.1- Operabilidad a través de teclado & 68,92 & 51 & 71,62 & 53 \\
\hline & 2.1.2- Posicionamiento del foco mediante teclado & 100 & 74 & 98,65 & 73 \\
\hline & 2.2.1- Ajuste del tiempo & 100 & 74 & 100 & 74 \\
\hline & 2.2.2- Acciónes para contenidos con movimiento & 100 & 74 & 100 & 74 \\
\hline & 2.3.1- Umbral de tres destellos & 100 & 74 & 100 & 74 \\
\hline & 2.4.1- Evitar bloques repetidos en varias páginas & 45,95 & 34 & 58,11 & 43 \\
\hline & 2.4.3- Posicionamiento secuencial del foco & 16,22 & 12 & 15,57 & 13 \\
\hline & 2.4.5- Múltiples caminos de navegación & 100 & 74 & 100 & 74 \\
\hline & 2.4.7- Foco visible & 77,03 & 57 & 74,32 & 55 \\
\hline \multirow{8}{*}{ P3 } & 3.1.1- Idioma de la página & 2,7 & 2 & 1,35 & 1 \\
\hline & 3.1.2- Idioma de las partes de la página & 100 & 74 & 100 & 74 \\
\hline & 3.2.1- Cambios al recibir el foco & 100 & 74 & 100 & 74 \\
\hline & 3.2.2- Cambios al recibir una entrada & 78,38 & 58 & 79,73 & 59 \\
\hline & 3.2.3- Coherencia en la navegación & 100 & 74 & 100 & 74 \\
\hline & 3.2.4- Identificación de componentes de igual función & 100 & 74 & 100 & 74 \\
\hline & 3.3.3- Sugerencias ante errores & 1,35 & 1 & & \\
\hline & 3.3.4- Prevención de errores & 1,35 & 1 & & \\
\hline P4 & 4.1.2- Nombre, función y valor & 56,76 & 42 & 56,76 & 42 \\
\hline
\end{tabular}

Tabla V. Criterios que TAW califica como "na: no aplicable", porcentaje y número de universidades en las que se presenta

\begin{tabular}{|c|c|c|c|c|c|}
\hline \multirow[b]{2}{*}{ Criterio } & & \multicolumn{2}{|c|}{ Principal } & \multicolumn{2}{|c|}{ Estudiantes } \\
\hline & & na* & $\%$ & na* & $\%$ \\
\hline 1.2 .1 & Sólo audio y sólo vídeo (grabado) & 68 & 91,89 & 71 & 95,95 \\
\hline 1.2 .2 & Subtítulos (grabados) & 68 & 91,89 & 71 & 95,95 \\
\hline 1.2 .3 & Audiodescripción o Alternativa (grab.) & 68 & 91,89 & 71 & 95,95 \\
\hline 1.2 .4 & Subtítulos (en directo) & 68 & 91,89 & 71 & 95,95 \\
\hline 1.2 .5 & Audiodescripción (grabado) & 68 & 91,89 & 71 & 95,95 \\
\hline 1.3 .2 & Secuencia significativa & 5 & 6,76 & 5 & 6,76 \\
\hline 1.4 .2 & Control del audio & 68 & 91,89 & 74 & 100 \\
\hline 2.4 .6 & Encabezados y etiquetas & 10 & 13,51 & 6 & 8,11 \\
\hline 3.3 .1 & Identificación de errores & 9 & 12,16 & 15 & 20,27 \\
\hline 3.3 .2 & Etiquetas o instrucciones & 10 & 13,51 & 12 & 16,22 \\
\hline 3.3 .3 & Sugerencias ante errores & 10 & 13,51 & 15 & 20,27 \\
\hline 3.3 .4 & Prevención de errores (legales, datos, etc) & 10 & 13,51 & 15 & 20,27 \\
\hline
\end{tabular}

na: no aplicable 
A continuación se inició la revisión manual de los criterios para los que se recomendaba, y para los que el programa no pudo realizar el análisis automático. Esta revisión, larga y exhaustiva, se ha realizado con la ayuda de la extensión "Web Developer" 1.1.9 para el navegador Firefox, extensión que incluye funciones para facilitar la visualización de determinados elementos de la página que permanecen ocultos en un uso normal de la misma (Hojas de estilo CSS, opciones de los formularios, enlaces y direccionamiento de los mismos, atributos de las imágenes, etc.) (Lauke, 2005). Esta fase ha dado los siguientes resultados.

Entre los criterios de conformidad que requieren revisión, de forma parcial, encontramos: siete que son exigibles para el nivel de conformidad AA, tanto en la página principal como en la de estudiantes, y 15 y 13 respectivamente, para el nivel A.

Tras la comprobación de los criterios que TAW recomienda revisar, encontramos (Tabla VI):
- En la página principal: 14,68 \% de criterios que se cumplen; $40,03 \%$ en los que se encuentran errores; y 45,29 \% en los que no se puede aplicar el análisis.

- En la página de estudiantes: 17,15 \% de criterios que se cumplen; $41,26 \%$ con errores; y $41,59 \%$ en los que no es de aplicación el análisis.

Resultados de la revisión de los criterios para los que al programa le fue "Imposible realizar comprobación automática" (Tabla VII):

- Página principal: 9,67\% supera el criterio de conformidad establecido; 72,05 \% contiene errores; y al 18,28 \% no es aplicable el análisis, por no contener ese tipo de elementos.

- Página de estudiantes: 12,06 \% cumplen el criterio de conformidad; $69,85 \%$ en el que se detectan errores; y al $18,09 \%$ no es de aplicación el análisis.

Tabla VI. Resultado de la revisión en los ítems en los que TAW recomienda: "Requiere revisión manual"

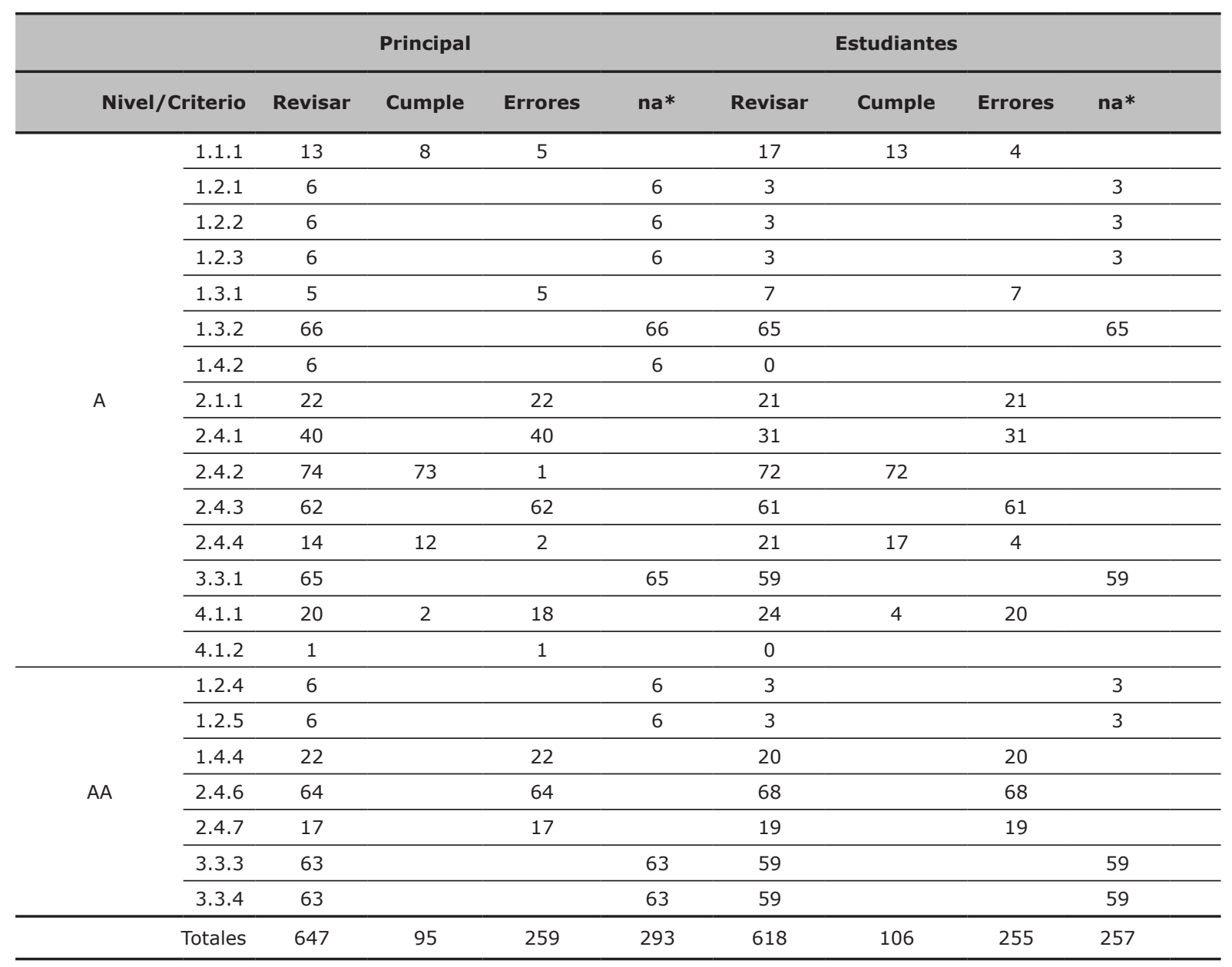

na: no aplicable 
Tabla VII. Resultado de la revisión en los ítems en los que TAW señala "Imposible realizar comprobación automática"

\begin{tabular}{|c|c|c|c|c|c|c|c|c|c|}
\hline & & \multicolumn{4}{|c|}{ Principal } & \multicolumn{4}{|c|}{ Estudiantes } \\
\hline \multicolumn{2}{|r|}{ Nivel/Criterio } & Revisar & Cumple & Errores & na* & Revisar & Cumple & Errores & na* \\
\hline \multirow{13}{*}{$A$} & 1.3 .3 & 74 & & 74 & & 74 & & 74 & \\
\hline & 1.4 .1 & 74 & & & 74 & 74 & & & 74 \\
\hline & 2.1 .1 & 51 & & 51 & & 53 & & 53 & \\
\hline & 2.1 .2 & 74 & & 74 & & 73 & & 73 & \\
\hline & 2.2 .1 & 74 & & & 74 & 74 & & & 74 \\
\hline & 2.2 .2 & 74 & & 74 & & 74 & & 74 & \\
\hline & 2.3 .1 & 74 & & & 74 & 74 & & & 74 \\
\hline & 2.4 .1 & 34 & & 34 & & 43 & & 43 & \\
\hline & 2.4 .3 & 12 & & 12 & & 13 & & 13 & \\
\hline & 3.1 .1 & 2 & 2 & & & 1 & 1 & & \\
\hline & 3.2 .1 & 74 & 44 & 30 & & 74 & 48 & 26 & \\
\hline & 3.2 .2 & 58 & & 58 & & 59 & & 59 & \\
\hline & 4.1 .2 & 42 & & 42 & & 42 & 2 & 40 & \\
\hline \multirow{10}{*}{$\mathrm{AA}$} & 1.4 .3 & 74 & 32 & 42 & & 74 & 41 & 33 & \\
\hline & 1.4 .5 & 74 & & 74 & & 74 & & 74 & \\
\hline & 2.4 .5 & 74 & 14 & 60 & & 74 & 17 & 57 & \\
\hline & 2.4 .7 & 57 & & 57 & & 55 & & 55 & \\
\hline & 3.1 .2 & 74 & & 74 & & 74 & & 74 & \\
\hline & 3.2 .3 & 74 & 4 & 70 & & 74 & 6 & 68 & \\
\hline & 3.2 .4 & 74 & 22 & 52 & & 74 & 33 & 41 & \\
\hline & 3.3 .3 & 1 & & & 1 & & & & \\
\hline & 3.3 .4 & 1 & & 1 & & & & & \\
\hline & Totales & 1220 & 118 & 879 & 223 & 1227 & 148 & 857 & 222 \\
\hline
\end{tabular}

na: no aplicable

Una vez computados los resultados del análisis automático y los de la revisión manual, los clasificamos en tres bloques: a) criterios que se cumplen, b) criterios con errores $y, c)$ criterios no evaluables (na) (Tabla VIII).

a) Encontramos 14 criterios (de 38) que se cumplen, aunque ninguno de ellos en el 100 $\%$ de universidades. En la página principal el porcentaje oscila entre el $98,65 \%$ del criterio 2.4.2 (en 73 de las 74 universidades); y el $4,05 \%$ del criterio 1.3.2 (en 3 de ellas). En la de estudiantes el porcentaje de cumplimiento oscila entre el $97,30 \%$ del criterio 2.4 .2 (en 72 de las 74 universidades); y el 4,05 \% del criterio 4.1.2 (en 3 de ellas).

b) Criterios que contienen errores: en esta situación se halla el $100 \%$ de los evaluables y ocurre, además, en un número alto de universidades, tanto en la página principal como en la de estudiantes (con la excepción del criterio 2.4.2).

c) Encontramos 15 criterios a los que no es de aplicación el análisis, destacando que en el $86,66 \%$ de los casos la "no aplicabilidad" de la norma incide en el $100 \%$ de ellos.
Atendiendo a los cuatro principios con sus respectivas pautas y criterios, encontramos:

En cuanto al principio 1 (P1: Perceptible):

- El criterio 1.1.1, único de la Pauta 1.1 (todo contenido no textual debe tener una alternativa textual), se cumple en las páginas principal y de estudiantes de $9(12,16 \%)$ y 14 $(18,92 \%)$ universidades respectivamente, y presenta errores en el resto.

- La Pauta 1.2 tiene un objetivo similar a la anterior, pero con los medios tempodependientes (audio, vídeo, subtítulos, etc.). Ninguno de los criterios de ésta es de aplicación, por tanto no provoca errores de accesibilidad.

- De los tres criterios de la Pauta 1.3 (secuencia de contenidos y de sus relaciones para no perder información); se cumplen el 1.3.1 en la página principal de 18 universidades $(24,32$ $\%)$, y en la de estudiantes de $14(18,92 \%)$; y el 1.3 .2 en $3(4,05 \%)$ y $4(5,40 \%)$ de ellas, no siendo de aplicación en el resto. El punto 1.3 .3 presenta errores en el $100 \%$ de universidades.

- La Pauta 1.4, formada por cinco criterios (separación entre primer plano y fondo en ima- 
Tabla VIII. Cómputo de resultados de la revisión automática y manual, criterios de las prioridades $A$ y $A A$ que se cumplen en su totalidad o en parte, los que contienen errores y en los que no es de aplicación (na); número de universidades a las que afectan

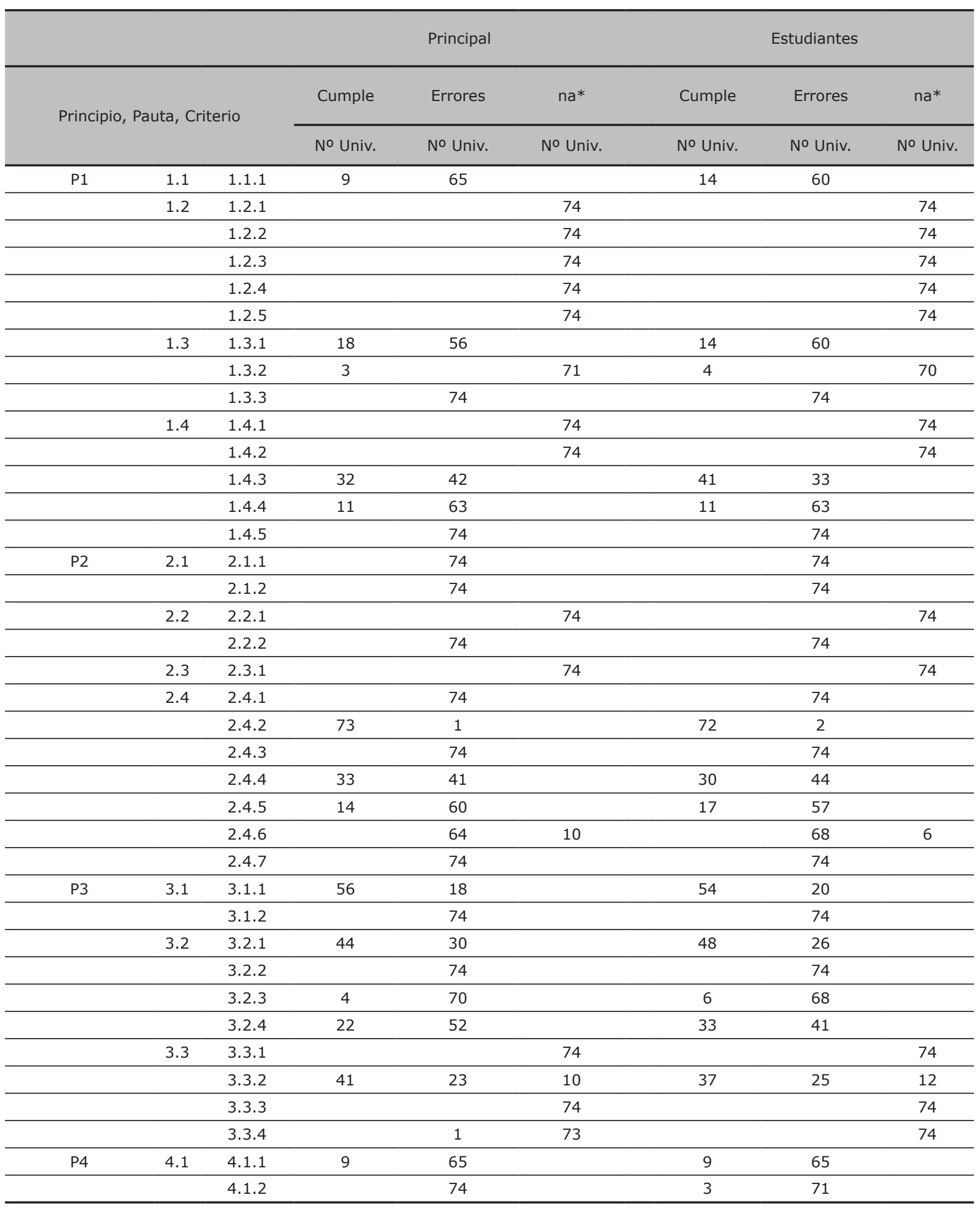

na: no aplicable 
gen, sonido o texto), se cumplen dos (relación de contraste entre texto y fondo y posibilidad de ampliación de texto), en parte de las universidades: el 1.4.3, en la página principal de $32(43,24 \%)$ y en la de estudiantes de $41(55,40 \%) ; y$ el 1.4 .4 , en ambas páginas de 11 de ellas $(14,86 \%)$. Otros dos $(1.4 .1 \mathrm{y}$ 1.4.2) no son de aplicación, y el resto presenta errores.

El análisis del Principio 2 (P2: Operable) revela los siguientes datos:

- La Pauta 2.1 (acceso mediante teclado a todas las funciones de las páginas), presenta errores en la totalidad de las universidades.

- Los dos criterios de la Pauta 2.2 (control del tiempo, actualización y carga de contenidos) se reparten entre la no aplicación del análisis (2.2.1) y los errores del criterio 2.2.2, en el $100 \%$ de la muestra.

- Para el único criterio de la Pauta 2.3, no es de aplicación el análisis.

- En la Pauta 2.4 (ayudas a la navegación y las búsquedas), se cumplen, en parte, tres de los siete criterios. El 2.4.2 (titulado de las páginas), es el de mayor cumplimiento: página principal de 73 universidades $(98,65 \%)$ y página de estudiantes de 72 de ellas $(97,30$ $\%$ ). Los criterios 2.4 .4 y 2.4 .5 (claridad en los enlaces y vías de navegación) se cumplen en la página principal de 33 y 14 universidades respectivamente, y en la de estudiantes de 30 y 17 de ellas; presentando errores el resto de criterios en la mayor parte de la muestra.

En el Principio 3 (P3: Comprensible) encontramos los siguientes resultados:

- En la Pauta 3.1 (los contenidos textuales deben ser legibles y comprensibles), el criterio 3.1.1 (idioma de la página) se cumple en la página principal de 56 universidades $(75,67 \%$ ) y en la de estudiantes de $54(72,97 \%)$. El otro criterio (3.1.2 - idioma de las partes) presenta errores en la totalidad de la muestra.

- La Pauta 3.2 (las operaciones que realizamos sobre una página deben ser predecibles), se cumple, en parte, en tres de los cuatro criterios que la forman, aunque en menos de la mitad de las universidades. Esta pauta presenta errores en todos sus criterios.

- Finalmente, la Pauta 3.3 (ayuda para evitar y corregir errores), se muestra como "no aplicable" en la totalidad de la muestra en tres de los criterios (3.3.1, 3.3.3 y 3.3.4), mientras que el 3.3.2 (instrucciones o etiquetas para la introducción de datos) se cumple en la página principal de $41(55,40 \%)$, y en la de estudiantes de 37 (50\%); presenta errores en la página principal de 23 y en la de estudiantes de 25; no es de aplicación en las restantes.
El Principio 4 (Robusto) y su Pauta 4.1 (hacer compatible el contenido de la página con otras aplicaciones y con posibles ayudas técnicas), contiene sólo dos criterios: el primero (4.1.1) se cumple en las dos páginas analizadas de nueve universidades $(12,16 \%)$ y presenta errores en el resto; mientras que el segundo (4.1.2) se cumple sólo en la página de estudiantes de tres universidades $(4,05 \%)$, presentando errores en el resto.

El resultado de la aplicación del análisis a las universidades españolas permite constatar (Tabla IX) que ninguna de ellas cumple al $100 \%$ con los requisitos de accesibilidad de la norma WCAG 2, observando que la horquilla de cumplimiento oscila entre el 65,79 \% de la de Granada, y el 36,84 \% de la Pontificia de Salamanca, en la página principal; y entre el 68,42\% de la de Granada, y el 36,84 de la de Navarra, en la página de estudiantes.

\section{DISCUSIÓN Y CONCLUSIONES}

El principal objetivo del presente trabajo ha sido determinar el nivel de accesibilidad de las páginas principales y de estudiantes de los sitios web de las distintas Universidades españolas de acuerdo con la norma WCAG 2.0. Los resultados encontrados, llevan a afirmar que los sitios web de las mismas siguen conteniendo diferentes errores que hacen difícil su acceso a diversos colectivos, al igual que señalaban estudios previos (Alba y otros, 2003; Ribera y otros, 2009; Rocha y otros, 2011; Rodríguezy otros, 2006; Térmens y otros, 2003; Térmens, 2002; Toledo, 2001). Aun con la precaución que supone comparar los resultados del presente estudio con éstos, puesto que en nuestro caso se utiliza una norma diferente de análisis (WCAG 2.0), más actual, precisa y verificable y, sobre la que poco se ha indagado en nuestro país; mientras que los estudios aludidos realizaron los análisis de acuerdo con la norma WCAG 1.0.

De los datos obtenidos podemos establecer una primera conclusión: después de revisar automática y manualmente los Principios, Pautas y Criterios de conformidad de la norma WCAG 2.0 (en sus niveles de prioridad $A$ y $A A$ ), se constata que ni una sola de las universidades españolas cumple con los requisitos de accesibilidad de dicha norma al $100 \%$, aunque la que mejor se encuentra y más se aproxima es la universidad de Granada, seguida de la de Alicante. Resultados que varían respecto a los hallados en estudios anteriores (que situaban en los primeros lugares universidades como la Politécnica de Cartagena, la de Sevilla o la de Oviedo) (Ribera y otros, 2009), que analizaban el nivel de accesibilidad de acuerdo con la norma WCAG 1.0.

En el cómputo global de resultados se observa que se supera un $13,12 \%$ de criterios en la página principal y un $13,12 \%$ en la de estudiantes. Se detectan errores en el 52,10\% de criterios en la página principal y en el $51,32 \%$ en la de estudiantes; mientras que el análisis no es aplicable en un $34 \%$ de criterios aproximadamente. 
Tabla IX. Listado alfabético de las universidades españolas con el porcentaje de cumplimiento de los criterios de conformidad WCAG 2, para los niveles A y AA, en las páginas analizadas

\begin{tabular}{|c|c|c|c|c|c|}
\hline \multirow{2}{*}{ Universidad } & $\%$ & $\%$ & \multirow{2}{*}{ Universidad } & \multirow{2}{*}{$\begin{array}{c}\% \\
\text { Entr.* }\end{array}$} & \multirow{2}{*}{$\begin{array}{c}\% \\
\text { Estud.* }\end{array}$} \\
\hline & Entr.* & Estud.* & & & \\
\hline A Coruña & 52,63 & 47,37 & Jaume I & 42,11 & 47,37 \\
\hline A Distancia de Madrid & 39,47 & 42,11 & La Laguna & 44,74 & 44,74 \\
\hline Abat Oliba & 44,74 & 47,37 & La Rioja & 44,74 & 44,74 \\
\hline Alcalá & 55,26 & 47,37 & Las Palmas Gran Canaria & 39,47 & 39,47 \\
\hline Alfonso X El Sabio & 42,11 & 44,74 & León & 55,26 & 57,89 \\
\hline Alicante & 60,53 & 63,16 & Lleida & 52,63 & 47,37 \\
\hline Almería & 50,00 & 52,63 & Málaga & 55,26 & 57,89 \\
\hline Antonio de Nebrija & 39,47 & 50,00 & Miguel Hernández & 52,63 & 50,00 \\
\hline Autónoma de Barcelona & 50,00 & 55,26 & Mondragon & 47,37 & 50,00 \\
\hline Autónoma de Madrid & 55,26 & 55,26 & Murcia & 55,26 & 42,11 \\
\hline Barcelona & 50,00 & 47,37 & Navarra & 42,11 & 36,84 \\
\hline Burgos & 50,00 & 52,63 & Oberta de Catalunya & 47,37 & 47,37 \\
\hline Cádiz & 50,00 & 52,63 & Oviedo & 47,37 & 44,74 \\
\hline Camilo José Cela & 39,47 & 44,74 & Pablo Olavide & 42,11 & 42,11 \\
\hline Cantabria & 47,37 & 47,37 & País Vasco & 52,63 & 50,00 \\
\hline Cardenal Herrera & 42,11 & 44,74 & Politécnica de Cartagena & 55,26 & 47,37 \\
\hline Carlos III de Madrid & 47,37 & 44,74 & Politécnica de Catalunya & 55,26 & 50,00 \\
\hline Castilla La Mancha & 52,63 & 52,63 & Politécnica de Madrid & 50,00 & 50,00 \\
\hline Católica de Ávila & 42,11 & 44,74 & Politècnica de València & 55,26 & 52,63 \\
\hline Católica de Valencia & 42,11 & 44,74 & Pompeu Fabra & 50,00 & 50,00 \\
\hline Católica San Antonio & 39,47 & 42,11 & Pontificia de Comillas & 39,47 & 42,11 \\
\hline Complutense de Madrid & 57,89 & 60,53 & Pontificia de Salamanca & 36,84 & 42,11 \\
\hline Córdoba & 47,37 & 44,74 & Pública de Navarra & 52,63 & 50,00 \\
\hline Deusto & 52,63 & 55,26 & Ramon Llull & 39,47 & 44,74 \\
\hline Europea de Madrid & 42,11 & 39,47 & Rey Juan Carlos & 50,00 & 50,00 \\
\hline Eur. Miguel de Cervantes & 42,11 & 47,37 & Rovira i Virgili & 50,00 & 57,89 \\
\hline Extremadura & 50,00 & 55,26 & Salamanca & 52,63 & 47,37 \\
\hline Francisco de Vitoria & 39,47 & 42,11 & San Jorge & 42,11 & 44,74 \\
\hline Girona & 42,11 & 44,74 & San Pablo & 39,47 & 42,11 \\
\hline Granada & 65,79 & 68,42 & Santiago Compostela & 52,63 & 55,26 \\
\hline Huelva & 44,74 & 47,37 & Sevilla & 55,26 & 55,26 \\
\hline IE Universidad & 39,47 & 42,11 & UNED & 55,26 & 47,37 \\
\hline Illes Balears & 57,89 & 57,89 & València & 50,00 & 42,11 \\
\hline Intern. de Andalucía & 39,47 & 39,47 & Valladolid & 50,00 & 47,37 \\
\hline Intern. de Catalunya & 50,00 & 50,00 & Vic & 44,74 & 47,37 \\
\hline Intern. Menéndez Pelayo & 44,74 & 55,26 & Vigo & 47,37 & 52,63 \\
\hline Jaén & 47,37 & 55,26 & Zaragoza & 47,37 & 52,63 \\
\hline
\end{tabular}

Entr.: Página principal o de entrada al sitio, Estud.: Página de estudiantes o alumnos 
Teniendo en cuenta los principios de manera separada, encontramos que el Principio 3 ("Comprensibilidad") es el que se cumple en un mayor porcentaje $(22,57 \%$ en la página principal y 24,05 en la de estudiantes), aunque si contemplamos de manera conjunta los criterios que se cumplen y los que no provocan problemas de accesibilidad (por no estar presentes sus contenidos), comprobamos que es el Principio 1 ("Perceptibilidad") el que presenta menos problemas de accesibilidad (en un $63,90 \%$ de la muestra, en la página principal, y un $64,86 \%$, en la de estudiantes).

El Principio 2 ("Operabilidad") es el segundo en porcentaje de criterios aprobados $(13,51 \%$ en la página principal y $13,40 \%$ en la de estudiantes), pero presenta un gran número de errores (en torno al $69 \%$ en cualquiera de las páginas).

El Principio 4 ("Robustez") es el que menos se cumple $(6,08 \%$ de universidades en la página principal y $8,10 \%$ en la de estudiantes), constatando que las mayores dificultades de accesibilidad se dan en la compatibilidad con aplicaciones y ayudas técnicas.

Si bien asumimos como limitación del estudio haber centrado el análisis en solo dos páginas (lo que podría considerarse insuficiente dado el gran número de páginas que incluyen algunos sitios web), sin embargo, es preciso resaltar que el hecho de tener que acceder a través de la página principal a los distintos lugares (en la mayoría de los casos), provoca que los posibles problemas de acceso en ella, determinen el acceso a las siguientes. A pesar de esta posible limitación, creemos que los resultados obtenidos, aplicando la norma WCAG 2.0, son novedosos y relevantes; al poner de relieve el gran número de errores detectados y el moderado cumplimiento de los requisitos de accesibilidad. Dada la repercusión que ello puede tener en el acceso a la web de personas con discapacidad, parece oportuno recomendar la necesidad de que los webmasters, encargados del diseño y mantenimiento de los sitios web de las universidades, hagan las adaptaciones oportunas para que estos sitios sean cada vez no sólo más atractivos y dinámicos; sino más cercanos y adaptados a cualquier persona que pretenda navegar a través de ellos, localizar la información o el conocimiento. Propuesta que se sostiene en las recomendaciones específicas acerca de la accesibilidad web, que defiende la conveniencia de que los contenidos estén accesibles para cualquier usuario, independientemente de sus condiciones personales o contexto de navegación (Greeff y Kotzé, 2009; Harper y Yesilada, 2008).

\section{BIBLIOGRAFÍA}

Alba Pastor, C.; Ruiz, N.; Zubillaga, A. (2003). Educación superior y discapacidad: accesibilidad de las páginas web de las universidades estatales. Comunicación y pedagogía: Nuevas tecnologías y recursos didácticos, (188), 25-31.
CITIC, Centro Tecnológico. (s. f.). TAW - Servicios de accesibilidad y movilidad web. Disponible en: http://www.tawdis.net/ [consulta: 23/02/2013].

Fundación Sidar. (s. f.). Pautas de Accesibilidad para el Contenido Web (WCAG) 2.0 Disponible en: http:// www.sidar.org/traducciones/wcag20/es/ [consulta: 20/02/2013].

García, J. (2006). Test de accesibilidad web (TAW). Boletic. Disponible en: http://www.astic.es/sites/ default/files/articulosboletic/mono4_3.pdf [consulta: 01/03/2013].

Greeff, M.; Kotzé, P. (2009). A lightweight methodology to improve web accessibility. Proceedings of the 2009 Annual Research Conference of the South African Institute of Computer Scientists and Information Technologists (pp. 30-39). Presentado en SAICSIT '09, New York, USA: ACM Press.

Harper, S.; Yesilada, Y. (Eds.). (2008). Web accessibility and guidelines. Web Accessibility: A Foundation for Research (pp. 61-78). New York: Springer.

Hassan Montero, Y.; Martín Fernández, F. J. (2004). Propuesta de adaptación de la metodología de diseño centrado en el usuario para el desarrollo de sitios web accesibles. Revista Española de Documentación Científica, 27 (3), 330-344.

Henry, S. L. (2002). Understanding Web Accessibility. En Constructing Accessible Web Sites. Birmingham, U.K.: Glasshaus.

Lauke, P. H. (2005). Evaluating Web Sites for Accessibility With Firefox | Ariadne: Web Magazine for Information Professionals. Disponible en: http:// www.ariadne.ac.uk/issue44/lauke [consulta: 28/02/2013].

Marquès, P. (s. f.). Internet, la entrada en una nueva era. Disponible en: http://peremarques.pangea. org/nuevaera.htm [consulta: 18/02/2013].

Pinto Martín, M. (2011). Desarrollo de un sitio web corporativo accesible usando Drupal. Disponible en: http://oa.upm.es/9304/ [consulta: 05/03/2013].

Ribera, M.; Térmens, M.; Frías, A. (2009). La accessibilidad de las webs de las universidades españolas. Balance 2001-2006. Revista Española de Documentación Científica, 32 (3), 66-88.

Rocha, R.; Cobo, Á.; Alonso, M. (2011). Administración electrónica en las universidades públicas españolas. Revista Española de Documentación Científica, 34 (4), 545-562.

Rodríguez, D. J.; Viera, J. G.; Castillo, J. (2006). Universidad y Accesibilidad. Situación en España. Revista Iberoamericana de Sistemas, Cibernética e Informática, 3 (2), 33-37.

SEDIC. (2011). Técnicas y herramientas para evaluar la accesibilidad web. Disponible en: http://www. sedic.es/autoformacion/accesibilidad/9-tecnicasherramientas.html [consulta: 28/02/2013].

Serrano Mascaraque, E.; Moratilla Ocaña, A.; Olmeda Martos, I. (2010). Métrica para la evaluación de la accesibilidad en Internet: propuesta y testeo. Revista Española de Documentación Científica, 33 (3), 378-396.

Térmens Graells, M.; Ribera Turró, M.; Sulé Duesa, A. (2003). Nivel de accesibilidad de las sedes web de 
las universidades españolas. Revista Española de Documentación Científica, 26 (1).

Térmens, M. (2002). La accesibilidad en las Web de las universidades andaluzas. XII Jornadas Bibliotecarias de Andalucía (pp. 213-225). Málaga: Asociación Andaluza de Bibliotecarios.
Toledo, P. (2001). La accesibilidad en las Web de las universidades andaluzas. Pixel-Bit: Revista de Medios y Educación, (17), 53-66.

W3C Working Group. (2006). Requirements for WCAG 2.0. Disponible en: http://www.w3.org/TR/wcag2req/ [consulta: 24/02/2013]. 